\title{
On the relation between two-dimensional and axisymmetric loads in plate flexure problems
}

\author{
Detlef Wolf \\ Department of Physics, University of Toronto, Toronto, Ontario, Canada, M5S 1 A 7
}

\section{Introduction}

The effect of the shape or strike length of loads on the deflection of thin elastic plates has been addressed by several authors (e.g. Brotchie and Silvester, 1969; Watts et al., 1975; Menke, 1981). In their discussions, the model of a two-dimensional load with a simple straight edge has, however, received little attention. This is even more remarkable in view of the simplicity of the solution for the deflection in this special case (e.g. Officer, 1974, p. 386; Jeffreys, 1976, p. 271), which permits the model's application in preliminary interpretations. The disregard of this model is probably a consequence of some uncertainty as to the scale of geological loads that is required in order that they be approximated by two-dimensional edges. Most authors have therefore preferred other elementary geometries, such as strips or disks, as first approximations of physical load distributions of appropriate shape (see Turcotte, 1979, for a recent review).

A notable exception is Walcott's (1970) interpretation of lithospheric flexure induced by the Laurentide ice sheet in Canada. In his analysis, Walcott regarded the extent of this load large enough that, for points close to its margin, it can be represented by a two-dimensional edge.

In this note, the solution appropriate to straightedged two-dimensional loads will be derived from the

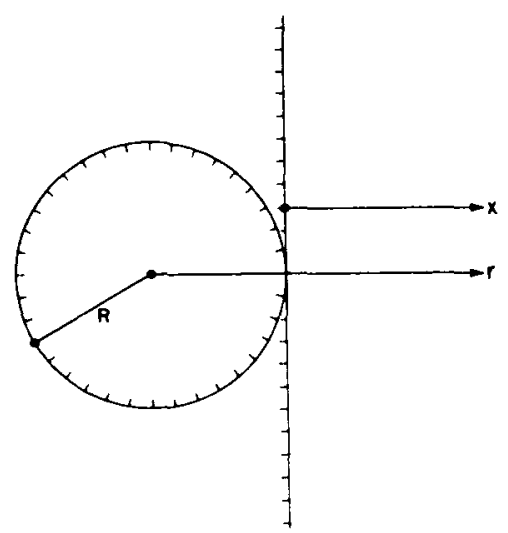

Fig. 1. Straight-edged axisymmetric load and its two-dimensional approximation more general solution for straight-edged axisymmetric loads (see Fig. 1). This limiting process will show that the accuracy of the two-dimensional approximation is controlled by both the scale of the load and the thickness of the elastic plate. Following that, several models appropriate to lithospheric flexure will be discussed.

\section{Theory}

The solution for the vertical surface displacement $w(r)$ due to a straight-edged axisymmetric unit load

$q(r)= \begin{cases}1, & 0 \leqq r<R \\ 0, & R<r<x\end{cases}$

of radius $R$, resting on a thin elastic plate of flexural scale length $1 / a$, has been given by Brotchie and Silvester (1969). If $\rho$ is the density of the inviscid substratum and $g$ denotes the acceleration due to gravity, the solution in terms of Bessel-Kelvin functions is

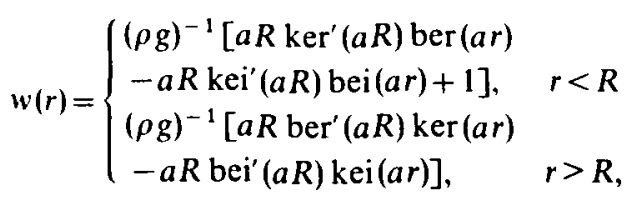

where a prime denotes derivation with respect to the argument and $r$ is the radial distance from the load axis. The flexural scale length $1 / a$ is related to the parameters of the plate and the substratum by

$a^{4}=\rho g / D$,

with $D$ the flexural rigidity of the plate. Introducing non-dimensional quantities $R^{*}=a R$ and $r^{*}=a r$. (2) can be written in the form

$w\left(x^{*}\right)= \begin{cases}(\rho g)^{-1}\left[R^{*} \operatorname{ker}^{\prime}\left(R^{*}\right) \operatorname{ber}\left(R^{*}+x^{*}\right)\right. & \\ \left.-R^{*} \operatorname{kei}^{\prime}\left(R^{*}\right) \operatorname{bei}\left(R^{*}+x^{*}\right)+1\right], & x^{*}<0 \\ (\rho g)^{-1}\left[R^{*} \operatorname{ber}^{\prime}\left(R^{*}\right) \operatorname{ker}\left(R^{*}+x^{*}\right)\right. & \\ \left.-R^{*} \operatorname{bei}^{\prime}\left(R^{*}\right) \operatorname{kei}\left(R^{*}+x^{*}\right)\right], & x^{*}>0,\end{cases}$

where $x^{*}=r^{*}-R^{*}$ is the non-dimensional distance from the edge of the disk. In order to reduce $(4)$ to the solution appropriate to two-dimensional loads, $R^{*}$ must be large, whereas $x^{*} / R^{*}$ must be small. From the asymp- 
totic approximations for Bessel-Kelvin functions for large arguments (McLachlan, 1934, pp. 168-172) we find

$\operatorname{ker}^{\prime}\left(R^{*}\right) \operatorname{ber}\left(R^{*}+x^{*}\right)$

$$
=-\frac{1}{2 R^{*}} \exp \left(x^{*} / \sqrt{2}\right) \cos \left(R^{*} / \sqrt{2}-\pi / 8\right)
$$$$
\left.\cdot \cos \left[\left(R^{*}+x^{*}\right) / \sqrt{2}-\pi / 8\right)\right] \text {, }
$$

$\operatorname{kei}^{\prime}\left(R^{*}\right)$ bei $\left(R^{*}+x^{*}\right)$

$$
=\frac{1}{2 R^{*}} \exp \left(x^{*} / \sqrt{2}\right) \sin \left(R^{*} / \sqrt{2}-\pi / 8\right)
$$

$$
\left.\cdot \sin \left[\left(R^{*}+x^{*}\right) / \sqrt{2}-\pi / 8\right)\right],
$$

$$
\operatorname{ber}^{\prime}\left(R^{*}\right) \operatorname{ker}\left(R^{*}+x^{*}\right)
$$$$
=\frac{1}{2 R^{*}} \exp \left(x^{*} / \sqrt{2}\right) \cos \left(R^{*} / \sqrt{2}+\pi / 8\right)
$$

$$
\left.\cdot \cos \left[\left(R^{*}+x^{*}\right) / \sqrt{2}+\pi / 8\right)\right],
$$

$$
\operatorname{bei}^{\prime}\left(R^{*}\right) \operatorname{kei}\left(R^{*}+x^{*}\right)
$$

$$
\begin{aligned}
= & -\frac{1}{2 R^{*}} \exp \left(x^{*} / \sqrt{2}\right) \sin \left(R^{*} / \sqrt{2}+\pi / 8\right) \\
& \left.\cdot \sin \left[\left(R^{*}+x^{*}\right) / \sqrt{2}+\pi / 8\right)\right] .
\end{aligned}
$$

Substitution in (4) yields

$w(x)= \begin{cases}(2 \rho g)^{-1}[2-\exp (a x / \sqrt{2}) \cos (a x / \sqrt{2})], & x<0 \\ (2 \rho g)^{-1} \exp (-a x / \sqrt{2}) \cos (a x / \sqrt{2}), & x>0,\end{cases}$

where $x=x^{*} / a$ has been used. This is the solution for a straight-edged two-dimensional load

$q(x)= \begin{cases}1, & x<0 \\ 0, & x>0 .\end{cases}$

Alternatively, (6) could have been derived directly from the two-dimensional form of the thin plate equation by matching the appropriate boundary conditions (e.g. Jeffreys, 1976, pp. 270-272).

In order to render $R^{*}$ sufficiently large for $(5)$ to be valid, $R$ or $a$ must be large enough. From (3) we realize that the latter is equivalent to requiring that $D$ be small. But $D=\mu h^{3} /[6(1-v)]$, where $\mu, l^{\prime}$ and $h$ denote Lamés second constant (rigidity), Poisson's ratio and elastic plate thickness, respectıvely. In a geophysical context, approximation (6) will therefore improve with increasing load diameter or decreasing lithospheric thickness. If $h=0$, we find that, for any value of $R$. $a \rightarrow \infty$ and therefore

$w(x)= \begin{cases}1 /(\rho g), & x<0 \\ 0, & x>0,\end{cases}$

which is the solution appropriate to local compensation.

\section{Numerical results}

In order to ascertain whether the two-dimensional solution (6) is suitable for approximating flexure in response to axisymmetric loads of finite radius, the non-
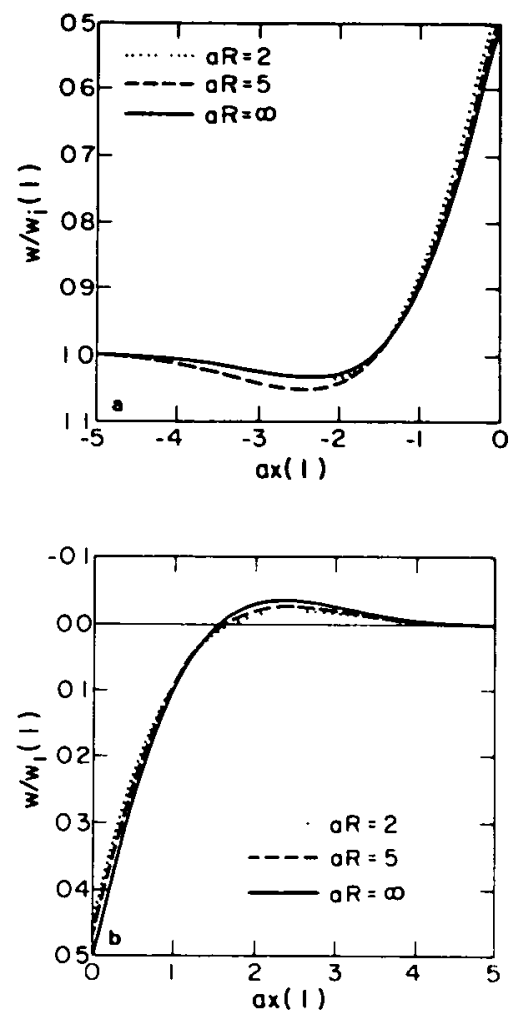

Fig. 2a and b. Normalized vertical displacement $w, w$ as a function of non-dimensional distance ax from the edge of the disk load for different non-dimensional radii $a R$ and a points below the load or $b$ beside the load. $w$, denotes the displacement for local compensation

dimensional disk solution (4) will be compared with the non-dimensional form associated with (6) for different values of $R^{*}=a R$.

Figure 2 compares axisymmetric solutions for nondimensional disk radii $R^{*}=2$ and $R^{*}=5$ with the appropriate two-dimensional solutions. In the area of positive deflection, the two-dimensional approximation can be considered good for $R^{*}>2$. This particularly applies to the steep slope near the edge of the load. In the range of the peripheral bulge the approximation deteriorates. Here displacements are generally small. and the absolute error increases to values of the order of the displacements for $R^{*}=2$.

For a demonstration of the geophysical significance of this. we consider disk loads of characteristic radii acting on elastic plates having thicknesses appropriate to the Earth's lithosphere. Here we will limit the analysis to $x>0$. This range contains the nodal point of the deflection curve and is therefore of particular interest. If necessary, the behaviour for $x<0$ can be inferred from Figure 1 after the model has been non-dımensionalized. To be specific we choose $\mu=0.67 \cdot 10^{11} \mathrm{Nm}^{-2}, \mathrm{v}=0.272$ and $\rho=3,380 \mathrm{~kg} \mathrm{~m}^{-3}$. This is fairly typical of the Earth at $100 \mathrm{~km}$ depth (Bullen. 1963. pp. 232-235). The load is taken to be $1 \mathrm{~km}$ thick with a density of $1 \mathrm{~kg} \mathrm{~m}$.

Figure 3 applies to $R=200 \mathrm{~km}$ and thin lithospheres. The configurations therefore have some relevance when modelling the flexure induced by Plesstocene Lake Bonneville (e.g. Nakıboglu and Lambeck. 1982) or 


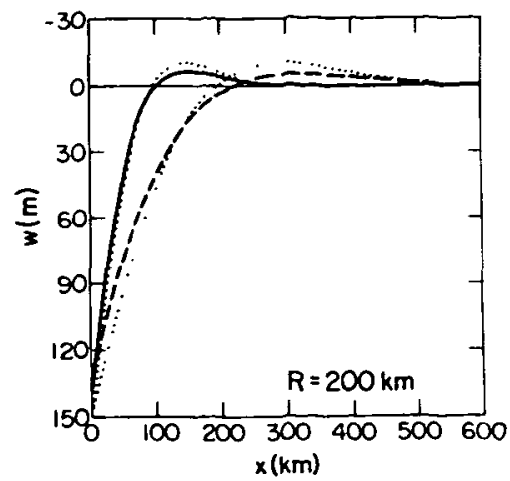

Fig. 3. Vertical displacement $w$ for a disk radius of $R$ $=200 \mathrm{~km}$ as a function of distance from the load edge for $h$ $=20 \mathrm{~km}$ or $a R=4.55$ (solid) and $h=50 \mathrm{~km}$ or $a R=2.29$ (dashed). The dotted lines delineate the appropriate two-dimensional solutions

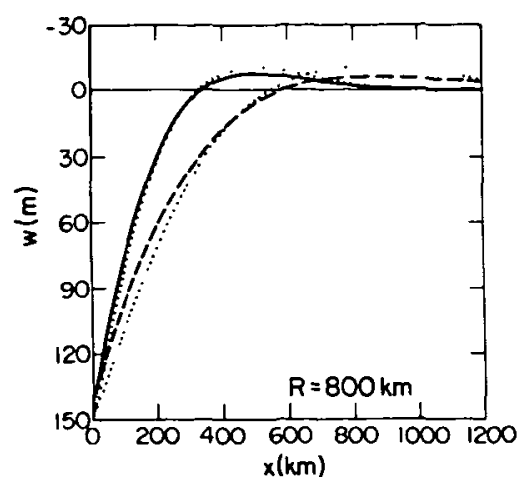

Fig. 4. Vertical displacement $w$ for a disk radius of $R$ $=800 \mathrm{~km}$ as a function of distance from the load edge for $h$ $=100 \mathrm{~km}$ or $a R=5.46$ (solid) and $h=200 \mathrm{~km}$ or $a R=3.24$ (dashed). The dotted lines delıneate the appropriate two-dimensional solutions

sedimentary basins (e.g. Haxby et al., 1976). If $h$ $=20 \mathrm{~km}$, the two-dimensional approximation is satisfactory, although the peripheral bulge is overestimated by more than 30 percent. For $h=50 \mathrm{~km}$, this misfit clearly exceeds 50 percent, and the inward shift of the nodal point becomes significant.

Figure 4 compares the solution for disk radii of $R$ $=800 \mathrm{~km}$ with the appropriate two-dimensional approximations. The scale of the load is fairly typical of the Fennoscandian ice sheet (e.g. Haskell, 1937). A lithospheric thickness of $100 \mathrm{~km}$ is conventional for continental regions; the enhanced value of $200 \mathrm{~km}$ reflects very recent estimates (e.g. Peltier, 1984). For $h$ $=100 \mathrm{~km}$, the two-dimensional approximation is good. except for the region of the peripheral bulge. If the thickness is $200 \mathrm{~km}$. the approximation deteriorates substantially and can be considered marginal at best.

In Figure 5 we have chosen $R=1.600 \mathrm{~km}$. The disk load may thus be taken as a crude approximation of the Laurentide ice sheet. If $h=100 \mathrm{~km}$, the model is similar to the one used by Walcott (1970). As can be seen, the two-dimensional approximation is very good and also holds in the peripheral region. For $h=200 \mathrm{~km}$,

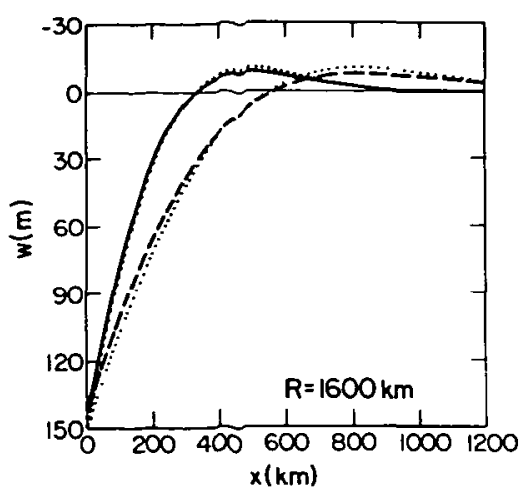

Fig. 5. Vertical displacement $w$ for a disk radius of $R=1,600$ $\mathrm{km}$ as a function of distance from the load edge for $h=100$ $\mathrm{km}$ or $a R=10.9$ (solid) and $h=200 \mathrm{~km}$ or $a R=6.49$ (dashed). The dotted lines delineate the appropriate two-dimensional solutions

the fit is still acceptable except for the peripheral bulge, which is overestimated by about 20 percent. It must be noted, however, that, for loads as extended as the Laurentide ice sheet, the Earth's sphericity is likely to become important. This problem is not followed up here but will be discussed in a separate investigation.

\section{Conclusions}

This note has attempted to clarify the relation between straight-edged axisymmetric loads and straight-edged two-dimensional loads by emphasizing the critical role of the elastic plate in this relation. It has been shown that loads of widely varying scale can be approximated by two-dimensional edges, provided that the lithosphere is sufficiently thin. As the distance from the edge of the load increases, the displacement $w$ decreases. This results in enhanced relative discrepancies between the two models in the region of the peripheral bulge. Whether this is significant when modelling data will ultimately depend on the magnitude of the errors associated with the measurements.

Acknowledgements. This investigation has been supported by a Natural Sciences and Engineering Research Council of Canada Postgraduate Scholarship. The figures have kindly been drafted by Khader Khan.

\section{References}

Brotchie, J.F., Silvester. R.: On crustal flexure. J. Geophỵs. Res. 74, 5240-5252, 1969

Bullen, K.E.: An introduction to the theory of seismology. 3rd edn. Cambridge: Cambridge University Press 1963

Haskell, N.A.: The viscosity of the asthenosphere. Am J. ScI. 33. 22-28, 1937

Haxby, W.F., Turcotte, D.L., Bird. J.M.: Thermal and mechanical evolution of the Michigan Basin. Tectonophysics 36. 57-75. 1976

Jeffreys. H.: The Earth, 6th edn. New York: Cambridge Unıversity Press 1976

McLachlan, N.W.: Bessel functions for engineers. Oxford Clarendon Press 1934 
Menke, W.: The effect of load shape on the deflection of thin elastic plates. Geophys. J. R. Astron. Soc. 65, 571-577, 1981

Nakiboglu, S.M., Lambeck, K.: A study of the Earth's response to surface loading with application to Lake Bonneville. Geophys. J.R. Astron. Soc. 70, 577-620, 1982

Officer, C.B.: Introduction to theoretical geophysics. Berlin: Springer-Verlag 1974

Peltier, W.R.: The thickness of the continental lithosphere. J. Geophys. Res. in press, 1984

Turcotte, D.L.: Flexure. Adv. Geophys. 21, 51-86, 1979
Walcott, R.I.: Isostatic response to loading of the crust in Canada. Can. J. Earth Sci. 7, 716-727, 1970

Watts, A.B., Cochran, J.R., Selzer, G.: Gravity anomalies and nexure of the lithosphere: A three-dimensional study of the Great Meteor Seamount, Northeast Atlantic. J. Geophys. Res. 80, 1391-1398, 1975

Received January 3, 1984; Revised version February 24, 1984 Accepted February 27, 1984 